\title{
Detecting Sybil Nodes in Wireless Sensor Networks using Two-hop Messages
}

\author{
Reza Rafeh ${ }^{1}$ and Mozhgan Khodadadi ${ }^{*}$ \\ 'Department of Computer Engineering, Arak University, Iran; r-rafeh@araku.ac.ir \\ 2Department of Computer Engineering, Malayer Branch, Islamic Azad University, Malayer, Iran; \\ khodadadi_mn@yahoo.com
}

\begin{abstract}
While there is a rapid growth of wireless sensor networks mustered in different areas such as military, agriculture, weather forecasting etc., an equal attention has also been paid on their security concern. A dangerous attack against these networks is Sybil attack in which one malicious node creates and propagates multiple fake identifiers. This attack mainly influences routing protocols and operations such as voting, data aggregation and reputation evaluation. In this paper we propose a distributed and efficient algorithm based on broadcasting two-hop messages to detect Sybil nodes in wireless sensor networks. In the proposed algorithm, by sending two-hop messages, each node finds its two-hop neighbors and the common neighbors between itself and each of its two-hop neighbors. The number of common neighbors is a good indicator to detect Sybil nodes. Experimental results show that the proposed algorithm outperforms similar existing algorithms with respect to true and false detection rates.
\end{abstract}

Keywords: Sensor Networks, Sybil Attacks, Tow-hop Messages

\section{Introduction}

Wireless sensor networks are a kind of ad hoc wireless networks, which have many usages in military, agriculture, health care, weather forecasting and so on. In order to fulfill their intended needs, such networks may use hundreds to thousands of tiny cheap sensor nodes. Considering the small size and low price of sensor nodes, some limitations emerge in terms of computational power, memory size, radio range and energy amount for these sensor nodes. Security of such network is a very important and is a challenge especially when they are employed in critical applications $\mathbf{s}^{1,2}$.

An important attack in wireless sensor networks is Sybil attack, in which an enemy captures a legal node or inserts an illegal node in the network. Then, this node which is called malicious node propagates several identifiers.

The enemy forges fake identifiers or duplicates existing nodes identifiers in different area of the network.
In this way, malicious node attracts a heavy traffic and considerably disrupts routing protocols. It even affects operations such as voting, date aggregation and reputation evaluation ${ }^{3-6}$.

Among solutions proposed for this attack is an algorithm based on radio source testing technique in which each node assigns an independent signaling channel to each of its neighbors ${ }^{7}$. In that research, another technique based on voting and registering nodes identifiers has been proposed to detect Sybil nodes. This technique is inefficient because of sensor nodes limitations. In addition, techniques which use voting cannot work effectively since some nodes are not reliable and may provide false information. On the other hand, identity authentication techniques ${ }^{8}$ usually need a huge memory space to save vital information of identity authentication (such as shared encryption keys, identity certificates, etc) being involved in processing complex checking algorithms. Algorithms based on received signal strength indicator (RSSI) ${ }^{9}$ like those outlined in ${ }^{12}$ cannot be a suitable solution because

${ }^{*}$ Author for correspondence 
radio signals are prone to be interfered with environment and, as a result, may affect accuracy of such algorithms.

In this paper we propose a distributed efficient algorithm to detect Sybil nodes in wireless sensor networks, resolving drawbacks of previous approaches. The proposed algorithm is based on broadcasting messages with 2-step time to live $(T T L=2)$ and needs no additional hardware (like GPS) or location-conscious nodes or locating nodes.

The rest of the paper is organized as follows: Section 2 reviews previous works. Sections 3 and 4 describe system assumptions and the proposed algorithm, respectively. Results of simulations are presented in Section 5. Finally, Section 6 concludes the paper.

\section{Related Works}

Sybil attacks were introduced for the first time in ${ }^{11}$ for peer-to-peer networks. $\mathrm{In}^{7}$, this attack was analyzed systematically for the first time for wireless sensor networks and mechanisms for confronting it were developed. These mechanisms include detection of Sybil nodes using radio source test, detection of Sybil nodes using randomized keys, confronting Sybil attacks using registration of identifiers and remote check of code or code verification. In algorithms which use radio source test technique, each node assigns a separate signaling channel to each of its neighbors. This technique cannot be efficient considering sensor node limitations. In order to detect Sybil nodes, identifier registration mechanism uses a central validation management unit and voting in the network.

An RSSI-based locating scheme has been introduced in $^{9}$ which uses proportion of RSSIs from multiple receivers in order to estimate nodes locations in the network. This mechanism has been used in ${ }^{12}$ to detect Sybil nodes. This scheme uses four location aware nodes (tracking nodes) capable of hearing packets throughout the network. Tracking nodes cooperate to locate any nodes sending packets. This is sufficient to detect Sybil nodes since all of them positioned in nearby locations.

$\operatorname{In}^{10}$, a technique has been provided to detect Sybil nodes which require no hardware or information on the number of nodes neighbors. This algorithm is distributed and needs no central mechanism like base stations or location aware nodes. $\mathrm{In}^{13}$ a new protocol has been proposed which uses identifier-based encryption. This protocol hardly allows nodes to acquire identifiers. This is used to confront Sybil attacks by not allowing malicious nodes to acquire multiple identifiers.
In $^{14}$ an RSSI-based algorithm has been proposed to detect Sybil attacks in sensor networks using the Leach protocol to perform clustering. Another mechanism has been provided by ${ }^{15}$, which uses an advanced RSSI-based technique to detect Sybil nodes while they are regulating their transfer powers. A new algorithm based on mechanism of Angle Of Arrival (AOA) detection named Trust Evaluation Base on AOA (TEBA) has been provided in $^{16}$. Taking into account that a Sybil node can create multiple identifiers, but has only one physical location, nodes whose signal phase differences are less than trust threshold (which is calculated by evaluating trust degrees for neighboring sensor nodes) are considered as Sybil nodes. In $^{17}$, a method has been provided to confront Sybil attacks, in which information on routes is collected by swarm intelligence algorithm during network activity and Sybil nodes are detected according to their energy changes when the network is active.

Like $^{14},{ }^{18}$ has provided an algorithm to detect Sybil attacks in sensor networks based on RSSI, which uses the Leach protocol to cluster the network. $\operatorname{In}^{19}$, another algorithm has been provided to detect Sybil nodes in Vehicular Ad hoc Networks (VANETs), which is also based on RSSI. $\operatorname{In}^{20}$ a new algorithm based on clients' puzzles and Learning Automata (LA) has been introduced to confront Sybil attacks in wireless sensor networks.

In general, disadvantages of existing algorithms for confronting Sybil attack are: high cost (need for GPS or nodes locations), no scalability, high rate of false detection and complexity of detection algorithms.

\section{System Assumptions and Attack Model}

A sensor network contains $\mathrm{N}$ sensor nodes randomly distributed within a 2-D space. Nodes are fixed and unaware of their own locations. The network is homogeneous (all network nodes have equal hardware /software facilities) and each node has a unique identifier. It is assumed that nodes are aware of network approximate density, $\mathrm{d}$, and that if the network density level changes, base station will inform the whole network securely. It is assumed that nodes communicate with each other via a wireless radio channel and use omni-directional broadcast. All nodes have fixed radio ranges equals to $\mathrm{r}$. Also, it is assumed that the sensor network is developing within a hostile environment, therefore, network is insecure and nodes may be captured by the enemy. 
Following the classification provided by ${ }^{7}$, in this paper we consider direct and simultaneous attack models with fake or stolen identifiers. The nodes captured by the enemy are called malicious nodes, and remaining nodes in the network are called normal nodes. Each malicious node propagates several identifiers (Sybil nodes). It is assumed that the number of nodes propagated by a malicious node is higher than the number of its normal neighbors ${ }^{10}$. And the last assumption is that Sybil nodes do not execute detection algorithm in order to save their energy and to prevent network normal nodes from becoming suspicious of their presence ${ }^{20}$.

\section{Proposed Algorithm}

Proposed algorithm is based on broadcasting two-hop messages whose structures are given in Figure 1. SenderID is the identifier of the sender node and TTL is the message time to live. Main idea of this algorithm is derived from the number of neighbors shared by two-hop neighboring nodes. Nodes $u$ and $v$ are two-hop neighbors if $r<d i s £ 2 r$, where this indicates the distance between $u$ and $v$. If the number of neighbors shared by two-hop neighboring nodes is above a given threshold, this may indicate the presence of Sybil nodes within common neighborhood.

According to most scenarios of wireless sensor networks, after distributing sensor nodes in the network environment, each node broadcasts "Hello" message in order to announce its presence. Then, each node $v$ discovers its direct (one-hop) neighbors and stores them in a vector named Neighbor-list.

Figure 2 illustrates radio range of two-hop neighboring nodes $u$ and $v$. Each node, like $u$ in Figure 2 becomes suspicious of Sybil attack in its neighborhood if it sees the

\section{SenderID} $T T L$

Figure 1. The structure of tow-hop messages.

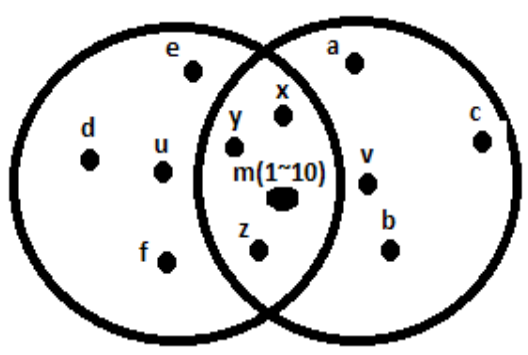

Figure 2. A topology of the network. number of its neighbor is exceeding the network density, $d$. Therefore, it creates and broadcasts a two-hop packet as $<<$ SenderID $=u, T T L=2>>$. Upon receiving this packet, each direct neighbor of $u$, e.g. nodes with identifiers $(1,2, \ldots 10, x, y, z, e, d, f, m)$ in Figure 2, initially, reduces one unit from its TTL field and, then, it is required to re-broadcast the packet because the field value is larger than zero (here, $T T L=1$ ). In this step, if the direct neighboring nodes of $u$ avoid re-broadcasting the received packet or they do not change its TTL field correctly, node $u$ considers them as malicious nodes.

Once the packet has been re-broadcasted by $u$ s direct neighbors, each of two-hop and one-hop neighboring nodes as well as node $u$ itself receive $k \geq 1$ packets containing $<<$ SenderID $=u, T T L=1>>$.

After looking at SenderID field of the received packet, node $u$ deletes the packet since the SenderID field equals its own identifier. Upon receiving the packet, one-hop neighbors (like $x, y, z$.e.d.f) compare its SenderID field with their own ones. Because the SenderID field differs from their own identifiers, one-hop neighbors search for the packet in Neighbor-list. When they find $u$ in their own Neighbor-list, they remove the received packet from their memory. Upon receiving this packet, two-hop neighbors (like $v, a, b, c$ ) store information in the packet including identifiers of the sender node (SenderID) and intermediate nodes or the common neighbors in a table called Common Neighbors Block (CNB). In other words, each node stores the list of its two-hop neighbors along with the set of intermediate nodes (or common neighbors) in its CNB table. From now on, we denote the CNB of $v$ by $\mathrm{CNB}_{v}$ in Figure 3. For example, if each node $u$ and $f$ generates and sends its own two-hop packet, node $v$, as one of their two-hop neighbors, updates its own $\mathrm{CNB}$ table, as shown in Figure 3. Next, node $v$ needs to refine its CNB table so that it contains only the set of suspect one-hop neighbors. Here, two refinement steps are considered.

\subsection{The First Refinement Step}

In this step, node $v$ traverses its $\mathrm{CNB}$ table to remove rows for which the number of intermediate nodes, namely the

\begin{tabular}{|c|c|}
\hline Two-Hop Neighbor & Intermediate_Nodes \\
\hline$u$ & $x, y, z, m, 1,2, \ldots, 10$ \\
\hline$f$ & $y, z, m, 1,2, \ldots, 10$ \\
\hline$\ldots$ & \\
\hline
\end{tabular}

Figure 3. The structure of CNB table. 
number of Intermediate-Node members, is below the threshold $T_{\max }$ because probably no Sybil node exists in this list. Otherwise, nodes existing in the list become suspected to be Sybil ones and need to be kept in the CNB table. The $T_{\max }$ threshold is calculated with respect to the area of radio range common between the two nodes located in each other's two-hop neighborhood like nodes $u$ and $v$ in Figure 2. In fact, this parameter indicates the maximum number of common neighbors expected to be between nodes $u$ and $v . T_{\max }$ calculation is provided in appendix A.

At the end of this step, each node $v$ in its CNB will have only the list of two-hop neighbors (and corresponding intermediate nodes) received from them more than $T_{\max }$ two-hop packets. Even CNB may become empty in the case the number of intermediate nodes is below $T_{\max }$ for all rows. Note that, in CNB, only nodes existing in Intermediate-Nodes section are suspected to be Sybil ones, not two-hop neighbors.

\subsection{The Second Refinement Step}

After the first refinement step, node $v$ can mark all intermediate nodes in its CNB as Sybil ones. But, as Figure 3 depicts, there may be a large number of normal nodes mistakenly marked as Sybil ones. This raises the rate of false detection. A possible solution for this problem is that adjacent nodes cooperate with each other in order to refine CNB more so that each node can detect Sybil nodes with its neighbors help. In this step, node $v$ sends its CNB to adjacent one-hop neighbors (nodes in Neighbors-List). In return, one-hop neighbors send their own CNBs to $v$. Sybil nodes, if any, broadcast empty CNBs because it was assumed that malicious Sybil nodes would not execute detection algorithm. This is a logical assumption, because it is not sensible for malicious nodes to execute detection algorithm and fall into its trap. Upon receiving its neighbors CNBs, node $v$ updates its CNB again, that is, in return for each two-hop neighbor (for example, node $u$ ) existing in its CNB, node $v$ begins to search for lists in $\mathrm{CNBs}$ it has received from neighboring nodes (e.g. node $a$ ) and, in the case $u$ exists in $\mathrm{CNB}_{a}$, if the intersection of intermediate nodes between $\langle v, u\rangle$ (that is, $\operatorname{CNB}_{\mathrm{v}}[u]$ [Intermediate-Nodes]) and $<a, u>$ (that is , $\mathrm{CNB}_{a}[u]$ [Intermediate-Nodes]) is $>=T_{\max }$, node $v$ considers the line corresponding to node $u$ in its own CNB equal to the intersection of these two sets. In other words, after receiving its neighbors CNBs like node $a$, node $v$ performs operations shown in Figure 4, repeating them for all CNBs it has received from its one-hop neighbors, and in this way, the second refinement step will be finished. Now, node $v$ marks all intermediate nodes in its CNB as Sybil ones.

\section{Efficiency Evaluation and Simulation Results}

In this section, we first evaluate the efficiency of the proposed algorithm in the form of memory overhead and energy consumption. Next, we provide simulation results of the proposed algorithm in the form of true and false detection rates and compare them with similar algorithms.

\subsection{Efficiency Evaluation}

\subsubsection{Memory Overhead}

Assuming that each node has $d$ neighbors on the average, the proposed algorithm needs $\mathrm{O}(d)$ space for each node to store its one-hop neighbors (Neighbor-list). Also, during executing of the proposed algorithm, each node needs to store its two-hop neighbors and intermediate nodes (CNB vector) requiring $\mathrm{O}\left(d^{2}\right)$ space. Of course, after executing the proposed algorithm, the space dedicated to $\mathrm{CNB}$ vector can be freed.

\subsubsection{Energy Consumption}

One important issue with sensor networks is the amount of energy consumed by each node because it is assumed that nodes batteries are not rechargeable. Since sending packets consumes much more energy than processing and receiving packets, one significant criteria for evaluating the efficiency of any algorithm developed for sensor

$$
\begin{aligned}
& \text { for each row } i \text { in } C N B_{v} \text {, if row } j \text { exists in } C N B_{a} \text { such that } \\
& \mathrm{CNB}_{v}[i]\left[T w o_{-} \mathrm{Hop}_{-} \text {Neighbor }\right]=\mathrm{CNB}_{a}[j]\left[\text { Tow } \text { Hop }_{-} \text {Neighbor }\right] \text { and } \\
& \mathrm{CNB}_{v}[i][\text { Intermediate_Node }] \mathrm{I} \mathrm{CNB}_{a}[j]\left[\text { Intermediate_Node }{ }^{3} T_{\max }\right. \text { then } \\
& \mathrm{CNB}_{v}[i][\text { Intermediate_Node }] \neg \mathrm{CNB}_{v}[i][\text { Intermediate_Node }] \text { I } \mathrm{CNB}_{a}[j][\text { Intermediate_Node }]
\end{aligned}
$$

Figure 4. Pseudo code of the second refinement step. 
networks is to calculate the number of sent packets imposed on the network due to using a given algorithm. In other words, this metric shows communication overhead. In the proposed algorithm, assuming that each node has $\mathrm{d}$ neighbors on the average, each node adjacent to Sybil nodes needs to broadcast a two-hop message to its neighbors and, also, needs to broadcast two-hop packets received from neighbors, if necessary. Finally, it needs to send its CNB list to adjacent neighbors in order to detect Sybil nodes.

Therefore, each node needs to send $1+d+d$ packets and, consequently, total communication overhead of the proposed algorithm is $\mathrm{O}(\mathrm{N} \times d)$ while the communication overhead of the algorithm in ${ }^{10}$ is $\mathrm{O}\left(N \times d^{2}\right)$.

\subsection{Simulation Results}

In order to evaluate the efficiency of proposed algorithm, some experiment were performed, result of which were compared with similar algorithms ${ }^{10,12,14,15,17-20}$. Evaluation metrics are true and false detection rates. The former is, in fact, a percentage of Sybil nodes being detected by a security algorithm and the latter is a percentage of normal nodes being detected mistakenly as Sybil ones by the security algorithm .

In our simulation, it is assumed that the network includes $N$ sensor nodes randomly distributed in a $100 \times 100 \mathrm{~m}^{2}$ area. The network contains $M$ malicious nodes with random distribution, each of which broadcasts $S$ fake identifiers. All nodes (normal and malicious) have the same radio range of $r=10 \mathrm{~m}$. Each simulation was repeated 500 times and the mean of 500 repetitions has been calculated.

\subsubsection{Experiment 1}

In this experiment, the efficiency of the proposed algorithm was evaluated in from of true detection rate. The number of malicious nodes was $M=10$; the number of Sybil identifiers broadcasted by each malicious node was $S=20$; and the total number of nodes was changed in range $150<N<500$ (with increment step of 50) and its effects on true detection rate of the proposed algorithm were evaluated.

Results are illustrated in Figure 5. As it is shown, detection rates are $80 \%, 85.14 \%$ and $92 \%$ for $N=150, N=200$, and $N=250$, respectively, while this metric is in rage $97 \%$ and $98.33 \%$ for $300<N<500$. Results of this experiment show that as network density increases (decreases), the proposed algorithm detection rate increases (decreases) because in a network with low density, fewer normal node $s$ lie adjacent to malicious ones and even there may be no normal nodes in their neighborhood which causes true detection rate decreases.

In contrast, as network density increases the number of normal nodes adjacent to malicious ones increases and they detect Sybil nodes more probably with each others help.

Figure 6 provides efficiencies of the proposed algorithm with similar algorithms in the from average true detection rates. In this experiment the number of malicious nodes was changed to $M>2$, the number of Sybil identifiers broadcast by each malicious node was changed to $S>14$ and the total number of nodes was in range 150 $<N<500$ (with incremental step of 50). As depicted in

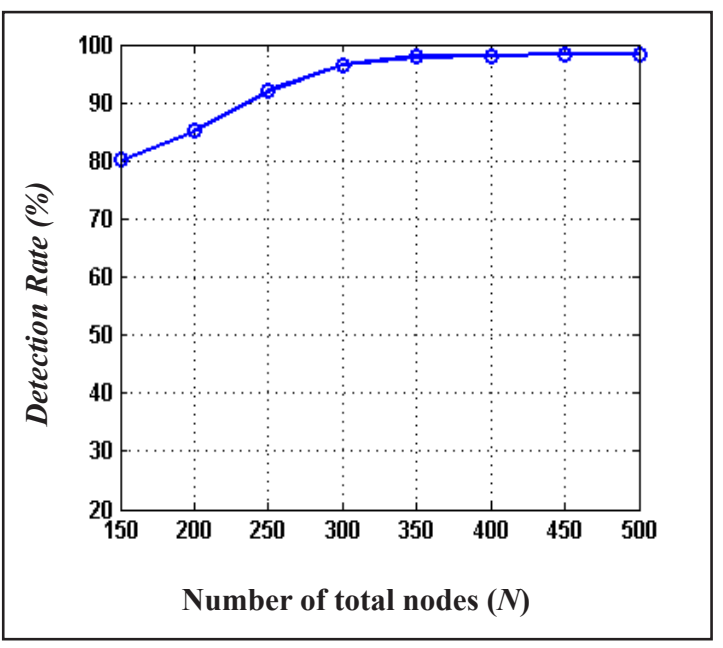

Figure 5. The effect of network density on detection rate of the proposed algorithm for $M=10$ and $S=20$.

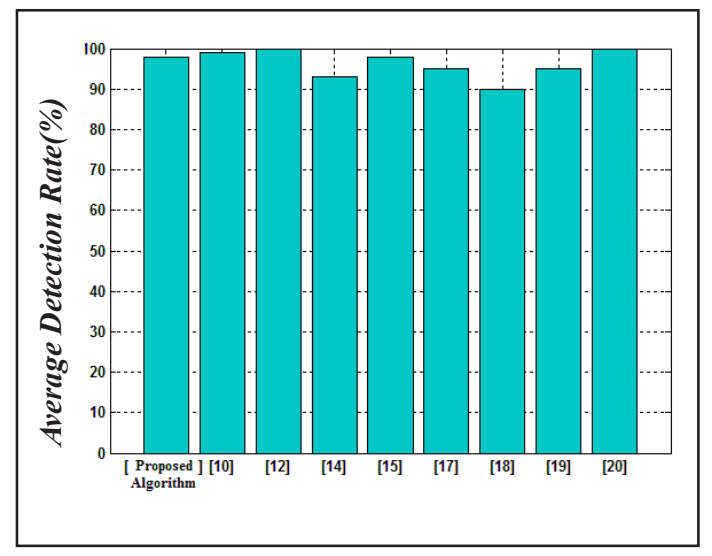

Figure 6. Comparing detection rate of the proposed algorithm with other algorithms. 
Figure 6, average rates of true detection are 99\%, 100\% and $100 \%$ for algorithms provided in $^{10,12}$ and $^{20}$, respectively, while this rate is $98 \%$ for the proposed algorithm. In ${ }^{12}$ RSSI has been used to locate nodes and if it is implemented in a real environment, its true detection rate will be lower than $100 \%$, because radio signals are prone to be in be interfered with environmental noises. True detection rates of algorithms provided in ${ }^{14,15,17-19}$ are $93 \%, 98 \%, 95 \%$, $90 \%$, and $95 \%$, respectively. Results of this comparison indicate favorable efficiency of the proposed algorithm in terms of true detection rate of Sybil nodes.

\subsubsection{Experiment 2}

The aim of this experiment was to evaluate the effect of the number of Sybil identifiers broadcasted by each malicious node on the rate of true detection of the proposed algorithm. In this experiment, the number of malicious nodes was $M=10$, the total number of nodes was $N=400$, and the number of Sybil identifiers broadcasted by each malicious node was in range $10<S<26$ (with incremental step of 2) effects of which on true detection rate of the proposed algorithm were evaluated. Results are depicted in Figure 7 which indicate that true detection rate increases (decreases) with an increase (decrease) in the number of Sybil identifiers because the proposed algorithm considers the number of neighbors shared by two nodes in two-hop neighborhood of each other. In other words, if the number of neighbors shared by two nodes which lie in two-hop neighborhood of each other exceeds a given threshold, it is more probable that Sybil nodes exist in the list of neighbors common between these two non-adjacent nodes. Thus, as the number of Sybil identifiers, $S$, increases, the probability executing the proposed algorithm by normal nodes (located in Sybil nodes neighborhood) increases and, as a result, true detection rate rises. Experimental results shown in Figure 7 support this matter. Detection rate of $90 \%$ and more than $98 \%$ are observed with $S=10$ and $S=18$, respectively.

\subsubsection{Experiment 3}

In this experiment, the effect of the number of malicious nodes, $M$, on true detection rate of the proposed algorithm was evaluated. The number of Sybil identifiers was $S=20$, the number of nodes was $N=400$, and the number of malicious nodes was in range $2<M<20$. The true detection rate of the proposed algorithm was evaluated and results are given in Figure 8.

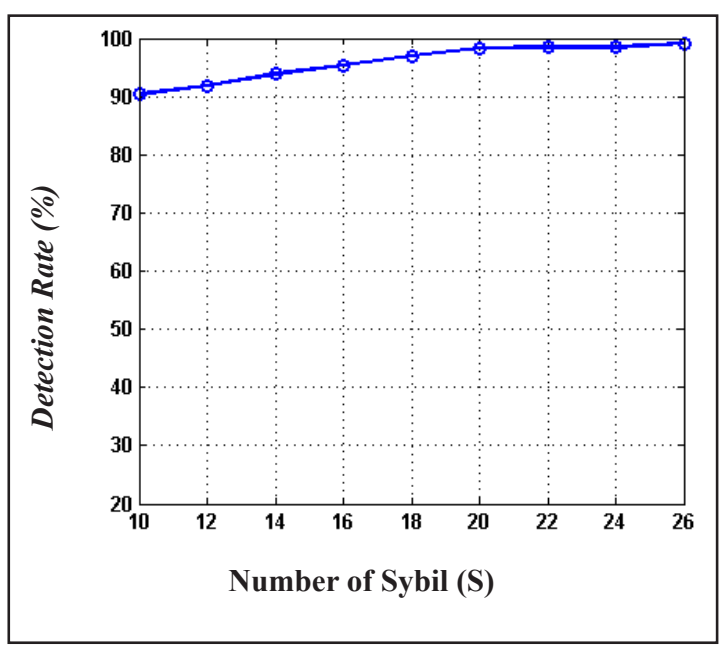

Figure 7. The effect of the number of Sybil identifiers on detection rate of the proposed algorithm for $M=10$ and $N=400$.

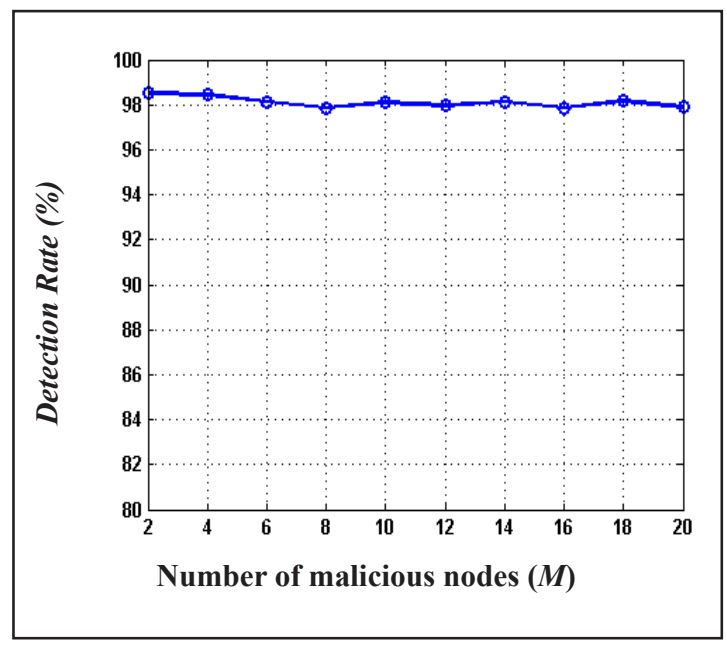

Figure 8. The effect of the number of malicious nodes on detection rate of the proposed algorithm for $S=20$ and $N=400$.

The results of this experiment indicate that changing the number of network malicious nodes has little effect on the true detection rate of the proposed algorithm, which is approximately $98 \%$. Since the proposed algorithm is completely distributed and each node detects Sybil nodes with the help of its one-and two-hop neighbors, increasing number of malicious nodes has no significant effect on the efficiency of the proposed algorithm in detecting Sybil nodes. This shows the scalability of the proposed algorithm. 


\subsubsection{Experiment 4}

The aim of this experiment was to evaluate the false detection rate of the proposed algorithm. In this experiment, the number of malicious nodes was $M=10$, the number of Sybil identifiers broadcasted by each malicious node was $S=20$, and total number of nodes was in range $150<N<500$ (with incremental step of 50). The false detection rate of the proposed algorithm and the algorithm proposed in ${ }^{10}$ are illustrated in Figure 9. Results show that the false detection rates in ranges $2 \%-3 \%$ and $2 \%-11 \%$ for the proposed algorithm and for algorithm in $^{10}$, respectively. When the network density is low (i.e., $N$ is less than or equal 250), the false detection rate of the proposed algorithm is $3 \%$ while this rate is about $2 \%$ for $N$ above 250 and below 500. For $N=500$, this rate becomes $3 \%$ again. This is because when the network density is low, there are fewer normal nodes adjacent to Sybil ones, therefore, fewer normal nodes cooperate to detect Sybil nodes which results in an increase in the false detection rate. Also, when the network density is high, more normal nodes lie very closely to Sybil ones, which may be detected by other normal nodes mistakenly as Sybil ones.

\subsubsection{Experiment 5}

This experiment was aimed at evaluating the effect of the number of Sybil identifiers broadcasted by each malicious node on false detection rate of the proposed algorithm. In this experiment, the number of malicious nodes was $M=10$, the number of nodes was $N=300$, and the number

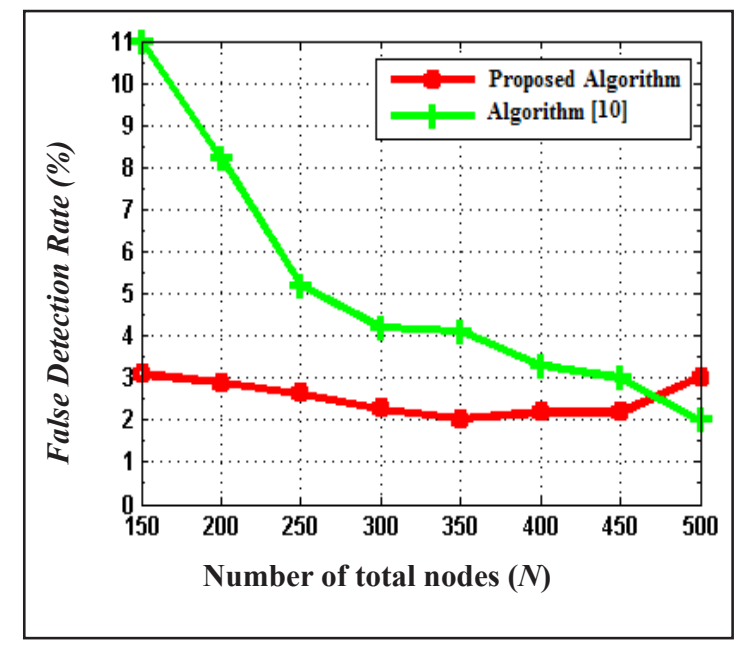

Figure 9. The effect of network density on false detection rate of the proposed algorithm and the algorithm in $^{10}$ for $M=10$ and $S=20$. of Sybil identifiers broadcasted by each malicious node was in range $10<S<26$ (with incremental step of 2). The result of comparing the proposed algorithm and that proposed in ${ }^{10}$ in terms of false detection rate is depicted in Figure 10. Results indicate that false detection rate of the proposed algorithm and of that in ${ }^{10}$ decrease (increase) as parameter $S$ (the number of Sybil identifiers) increases (decreases). However, the false detection rate of the proposed algorithm is lower than the algorithm $\mathrm{in}^{10}$. In the proposed algorithm, when $S=10$, the false detection rate is $12 \%$ and as $S$ increases, this rate decreases to the point where it is about $2 \%$ for $S>20$. Since the proposed algorithm, similar to the algorithm in ${ }^{10}$, uses neighborhood information (the number of adjacent nodes) to detect Sybil nodes, the number of Sybil identifiers broadcasted by each malicious node will have an effect on the efficiency of both algorithms.

\subsubsection{Experiment 6}

The aim of this experiment was to evaluate the effect of the number of malicious nodes, $M$, on false detection rate of the proposed algorithm. In this experiment, the number of Sybil identifiers was $S=20$, the number of nodes was $N=400$, and the number of malicious nodes was in range $2<M<20$ (with incremental step of 2). Results are depicted in Figure 11 and show that when there exist only two malicious nodes in the network, false detection rate is $0.7 \%$ which increases with an increase in $M$ so that this is about $3.5 \%$ for $M=20$. This is because for each malicious node in the network, it is possible that there may be some

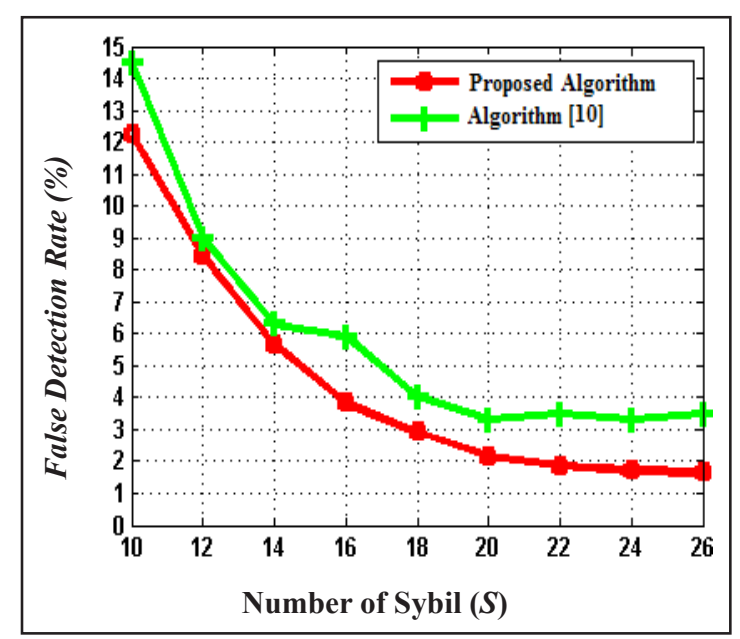

Figure 10. The effect of the number of Sybil identifiers on false detection rate of the proposed algorithm and the algorithm in ${ }^{10}$ for $M=10$ and $S=20$. 
normal nodes in its nearby neighborhood and may be mistakenly detected as malicious nodes. Thus, as the number of network malicious nodes increases, the false detection rate increases too.

Figure 12 compares the proposed algorithm with other algorithms in ${ }^{10,12,14,15,18,20}$ in terms of false detection rate. Among these algorithms only ${ }^{14}$ is slightly better than the proposed algorithm with $0.5 \%$ lower false detection rate. Of course, $\mathrm{in}^{14}$, a special case of Sybil attacks is considered, in which the malicious node tries to be selected as head-cluster in the LEACH clustering algorithm. Since the base station detects Sybil nodes, false detection rate is lower in this algorithm than in the proposed algorithm and other algorithms.

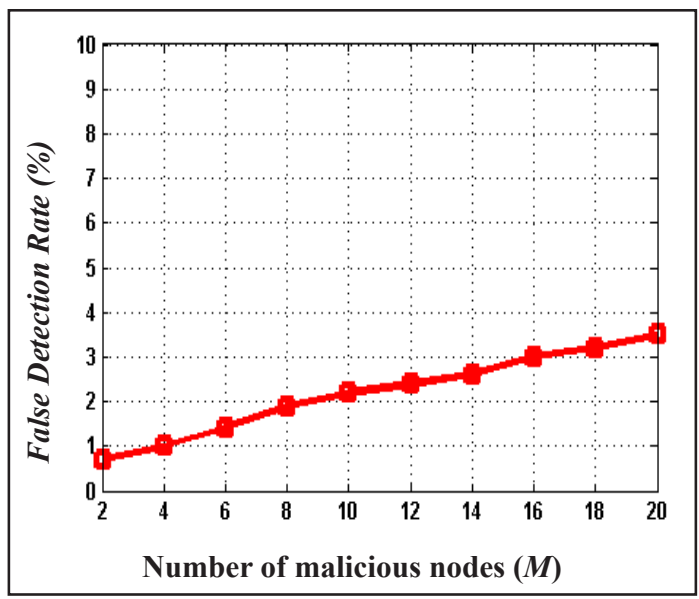

Figure 11. The effect of number of malicious nodes on false detection rate of the proposed algorithm $N=400$ and $S=20$.

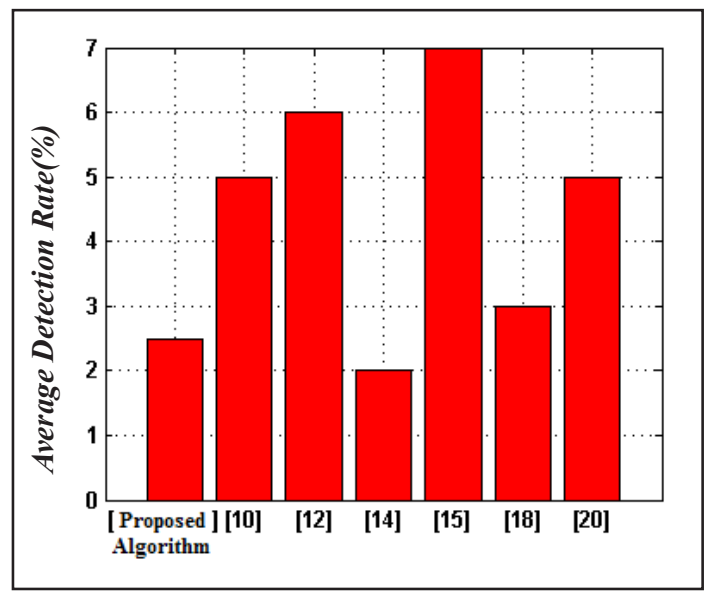

Figure 12. Comparing false detection rate of the proposed algorithm with other algorithms.

\section{Conclusion}

This paper presented a dynamic and distributed algorithm to detect Sybil nodes in wireless sensor networks. In this algorithm, nodes attempt to detect their neighboring Sybil nodes by broadcasting two-hop messages to their two-hop neighbors. The proposed algorithm has been simulated and its efficiency has been compared with other available algorithms. Experimental results indicate better performance of the proposed algorithm in viewpoints of true detection rate and false detection rate.

\section{References}

1. Akyildiz I.F, Su W, Sankarasubramaniam Y, Cayircl E. A survey on sensor networks. IEEE Communication Magazine. 2002 Aug; 40:102-14.

2. Akyildiz IF, Kasimoglu IH. Wireless sensor and actor networks: research challenges. Ad Hoc Networks. 2004; 2(4):351-67.

3. Karlof C, Wagner D. Secure Routing in Wireless Sensor Networks: Attacks and Countermeasures. AdHoc Networks. 2003 Sep; 1(2-3):299-302.

4. Walters JP, Liang Z, Shi W, Chaudhary V. Wireless Sensor Network Security: A Survey. Security in Distributed, Grid and Pervasive Computing. Yang Xiao, editors. Auerbach Publications, CRC Press; 2006.

5. Yick J, Mukherjee B, Ghosal D. Wireless sensor network survey. Computer Networks. 2008; 52:2292-330.

6. Padmavathi G, Shanmugapriya D. A survey of attacks, security mechanisms and Challenges in wireless sensor networks. IJCSIS. 2009 Aug; 4(1 \& 2):1-9.

7. Newsome J, Shi E, Song D, Perrig A. The Sybil Attack in Sensor Networks: Analysis and Defenses. International Symposium on Information Processing in Sensor Networks; 2004 Apr; New York. p. 259-68.

8. Liu D, Ning P. Establishing pairwise keys in distributed sensor networks, ACM Conference on Computer and Communications Security; 2003 Oct 27-30; New York. p. 52-61.

9. Zhong S, Li L, Liu YG, Yang YR. Privacy-preserving location based services for mobile users in Wireless Networks. Technical Report. YALEU/DCS/TR-1297, Yale Computer Science; 2004.

10. Ssu KF, Wang WT, Chang WC. Detecting Sybil attacks in wireless Sensor Networks using neighboring information. Proceedings of the Computer Networks. 2009; 53:3042-56.

11. Douceur JR. The Sybil attack. First International Workshop on Peer-to-Peer Systems (IPTPS ‘02); 2002 Mar 7-8; USA.

12. Demirbas M, Song Y. An RSSI-based scheme for Sybil attack detection in wireless sensor networks. IEEE Computer 
Society International Symposium on World of Wireless, Mobile and Multimedia Networks; 2006 Jun 23-29; Buffalo, New York. p. 570-74.

13. Butler K. Ryu S, Traynor P, McDaniel PD. Leveraging Identity-Based Cryptography for Node ID Assignment in Structured P2P Systems. TPDS. 2009; 20(12):1803-15.

14. Chen S, Yang G, Chen S. A Security Routing Mechanism against Sybil Attack for Wireless Sensor Networks. International Conference on Communications and Mobile Computing; 2010 Apr 12-14; Shenzhen.

15. Misra S, Myneni S. On Identifying Power Control Performing Sybil Nodes in Wireless Sensor Networks using RSSI. Global Telecommunications Conference; 2010 Dec 6-10; Miami, FL.

16. Zhang Y, Fan K-F, Zhang S-B, Mo W, AOA based trust evaluation sheme for Sybil attack detection in WSN. Journal on Application Research of Computers. 2010.
17. Muraleedharan R, Ye X, Osadciw LA. Prediction of Sybil Attack on WSN using Bayesian Network and Swarm Intelligence. Wireless Sensing and Processing; 2008 Mar; Orlando, FL, USA.

18. Jangra A, Swati, Priyanka. Securing LEACH Protocol from Sybil Attack using Jakes Channel Scheme (JCS), International Conferences on Advances in ICT for Emerging Regions(ICTer2011); 2011 Sep 1-2; Colombo.

19. Jiangtao W, Geng Y, Yuan S, Shengeshou C. Defending against sybil attacks based on received signal strength in wireless sensor networks. Journal of Electronics. 2008 Oct; 17 (4):611-14.

20. Jamshidi M, Esnaashari M, Meybodi MR. An Algorithm for Defending Sybil Attacks based on Client Puzzles and Learning Automata for Wireless Sensor Networks. 18th National Conference of Computer Society of Iran; 2013 Mar 14-16; Sharif University, Tehran, Iran.

\section{Appendix A: Calculation of $T_{\max }$ Threshold}

Assuming that each node's radio range is $r$, maximum region common between two-hop neighboring nodes $u$ and $v$ occurs when distance between them is $r+1$ (Figure A.1)). In order to obtain surface area of this common region, $\mathrm{A}(\mathrm{x})$, we can divide it into 4 equal parts (Figure A.2), then we calculate surface area of one part and multiply it by 4 .

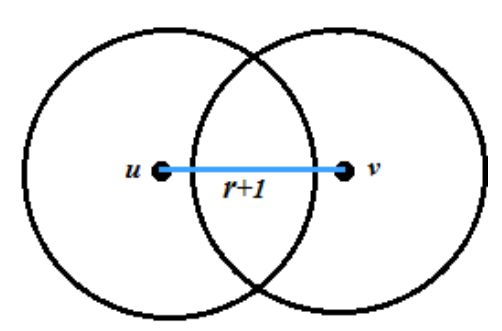

Figure A.1. Most common area between two-hop neighbor nodes

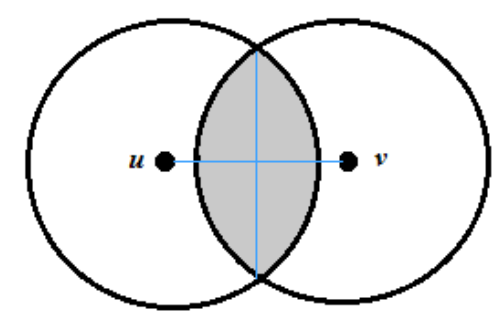

Figure A.2. Dividing common region into four equal regions.
The area of $B(x)$, as illustrated in Figure A.3, is given by:

$$
B(x)=\frac{q r^{2}}{2}, \quad q=\arccos \frac{æ r+1}{\grave{e}} \frac{\not}{2 r} \varnothing
$$

And, the area of $C(x)$, as illustrated in Figure A.4, is given by:

$$
C(x)=\frac{\frac{r+1}{2} \cdot \sqrt{r^{2}-\frac{æ r+1 \ddot{o}^{2}}{2} \varnothing}}{2}
$$

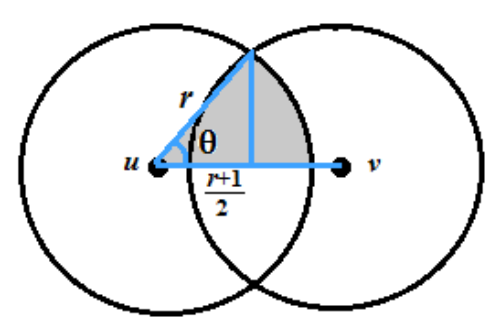

Figure A.3. Gray region, $B(x)$

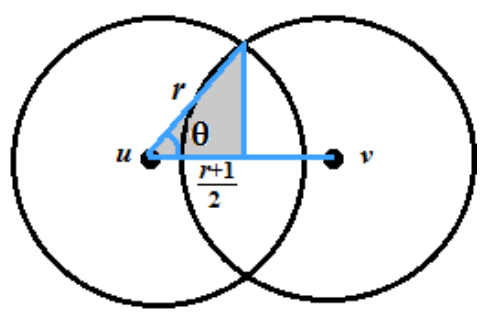

Figure A.4. Gray region, $C(x)$ 
And, The area of $D(x)$, as illustrated in Figure A.3, is given by:

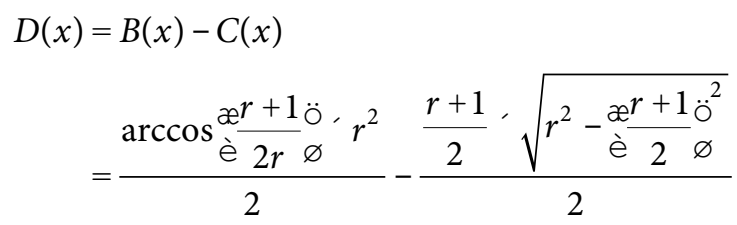

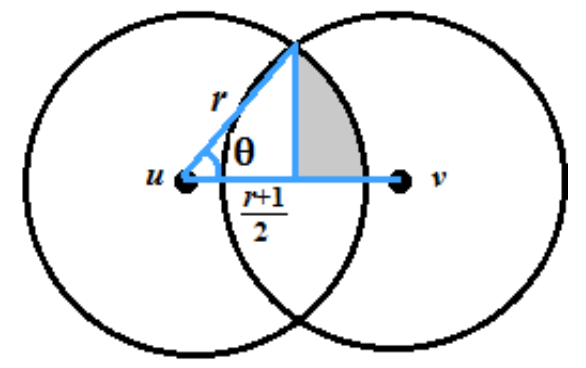

Figure A. 5. Gray region, $D(x)$
Finally, the total common region, as illustrated in Figure A.2, is equal:

$$
A(x)=4^{\prime} D(x)
$$

As result, $T_{\max }$ threshold is given by:

$$
T_{\max }=\frac{A(x)}{r^{2} \mathrm{p}} \cdot d
$$

where, $d$ is average number of node's neighbors. 